\title{
Brain Abscess Caused by Lactococcus Lactis in a Young Male
}

\author{
Intisar Ahmed ${ }^{1}$, Kashif Aziz $^{2}$, Hameed Tareen ${ }^{3}$ and Muhammad Arslan Ahmed ${ }^{4}$ \\ ${ }^{1}$ Department of Medicine, Section of Cardiology, The Aga Khan University, Karachi, Pakistan \\ ${ }^{2}$ Department of Medicine, Russel Hall Hospital, Pensnett Rd, Dudley, United Kingdom \\ ${ }^{3}$ Department of Medicine, Section of Nephrology, The Aga Khan University, Karachi, Pakistan \\ ${ }^{4}$ Department of Medicine, The Aga Khan University, Karachi, Pakistan
}

\begin{abstract}
Lactococcus lactis cremoris is one of the gram positive cocci, not known to be pathogenic in humans. We report a case of brain abscess due to lactococcus lactis in an adolescent. An 18-year male with congenitally corrected transposition of great arteries and dextrocardia was admitted with fever, headache and right-sided numbness. Magnetic resonance imaging revealed a well circumscribed irregular heterogeneous abnormal signal intensity lesion in left temporo-parietal lobe having central area of diffusion restriction and peripheral wall enhancement on post-contrast images. He underwent mini-craniotomy for abscess drainage. Pus culture revealed growth of lactococcus lactis. He was treated with ceftriaxone and remained disability-free on six month follow-up. To our knowledge, this is one of the few reports of brain abscess caused by lactococcus lactis.
\end{abstract}

Key Words: Brain abscess, Lactococcus lactis, Adolescent.

How to cite this article: Ahmed I, Aziz K, Tareen H, Ahmed MA. Brain Abscess Caused by Lactococcus Lactis in a Young Male. J Coll Physicians Surg Pak 2021; 31(07):852-854.

\section{INTRODUCTION}

Lactococcus lactis is a spherical-shaped, gram-positive bacterium used widely for production of dairy products such as milk, cheese and yogurt. ${ }^{1}$ Due to its important applications, simple metabolism and limited biosynthetic capabilities, its genome has been sequenced to help researchers understand genes that are involved in fermentation pathway. ${ }^{2}$ Lactococcus lactis is also found on plants and within the digestive system of cattle. It is believed that, in nature, lactococcus lactisstays inactive on plant surfaces awaiting to be ingested by animals, where it becomes active and multiplies in gut. ${ }^{3}$ This bacterium is commonly considered to be non-pathogenic; however, human infections have been reported. It has been reported as the causative agent for liver abscesses, infective endocarditis and paraphyrngeal abscesses..$^{4-7}$ Main risk factor has been intake of unpasteurized milk and plant products through oropharyngeal route. ${ }^{6}$ Central nervous system infection is very rare and only a few cases of brain abscess have been reported. ${ }^{7-9}$ To our knowledge, this is one of the few cases of brain abscess, secondary to lactococcuslactis.

Correspondence to: Dr. Intisar Ahmed, Department of Medicine, Section of Cardiology, The Aga Khan University Karachi, Pakistan

E-mail: intisarahmed87@gmail.com

Received: October 10, 2019; Revised: November 19, 2019;

Accepted: November 27, 2019

DOI: https://doi.org/10.29271/jcpsp.2021.07.852

\section{CASE REPORT}

An 18-year male of south Asian descent with congenitally corrected transposition of great arteries, presented with one week history of fever documented up to $102^{\circ} \mathrm{F}$, headache and right-sided numbness. Headache was episodic and severe in intensity. Other concern was his impaired feeling on right side. He described that he felt numb on right side of the body and face. This was causing impairment in his daily routine, so he came to hospital for treatment. He had no such symptoms previously. This was preceded a week ago by severe left ear ache, treated as otitis media, by a general practitioner with symptomatic resolution. He had no history of prior hospital admission. He was living with his family at his father's house and was mobile. Although before this disease, he was riding bicycle. But for the last one week, due to numbness, he was hesitant to ride the bicycle. He did not report any impairment in balance, vertigo, neglect of one side of body; and there was no history of fits. On presentation to us, he was afebrile and hemodynamically stable.

On neurological examination, he had a Glasgow coma scale of $15 / 15$ with intact higher mental functions and no signs of meningeal irritation. There was sensory loss in all three trigeminal areas on right side, i.e., ophthalmic, maxillary and mandibular, with reduced touch and temperature sensations without motor deficit. He had decreased tactile sensation on right arm. Rest of the sensory and motor examination was unremarkable with bilaterally downgoing plantars and an absence of signs of cerebellar disease. On cardiovascular examination, 
there was right-sided apex beat, and pan-systolic murmur due to ventricular septal defect.

Baseline investigations revealed haemoglobin of $181 \mathrm{~g} / \mathrm{l}(123$ $\mathrm{g} / \mathrm{l}-166 \mathrm{~g} / \mathrm{l})$ and platelets of $121 \times 10^{9} / \mathrm{l}\left(154 \times 10^{9} / \mathrm{l}-433 \times 10^{9} / \mathrm{l}\right)$ with a normal white cell count and erythrocyte sedimentation rate (ESR) of $1 \mathrm{~mm} / 1^{\text {st }}$ hour. Blood cultures and malarial parasite smear were negative. Magnetic resonance Imaging (MRI) brain with contrast revealed "well-circumscribed irregular shaped heterogeneous abnormal signal intensity lesion in left temporo-parietal lobe with significant surrounding brain oedema, causing mass effect and midline shift towards right side". There was central area of diffusion restriction with peripheral wall enhancement on post-contrast images (Figures 1 and 2).

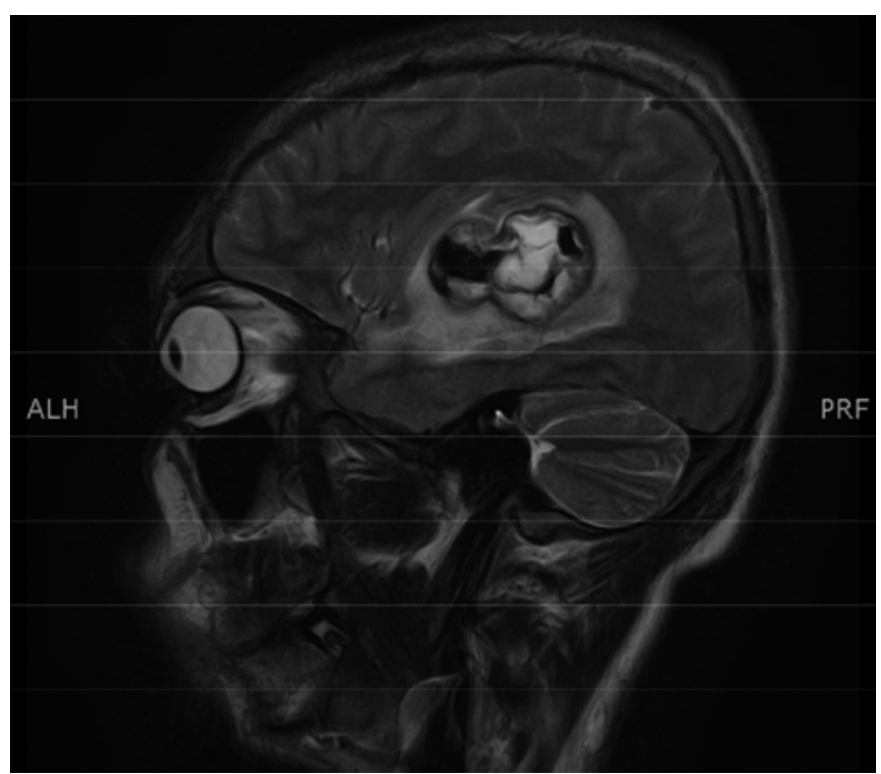

Figure 1: MRI brain T2 sequence sagittal view. Brain abscess with surrounding oedema in left temporo-parietallobe.

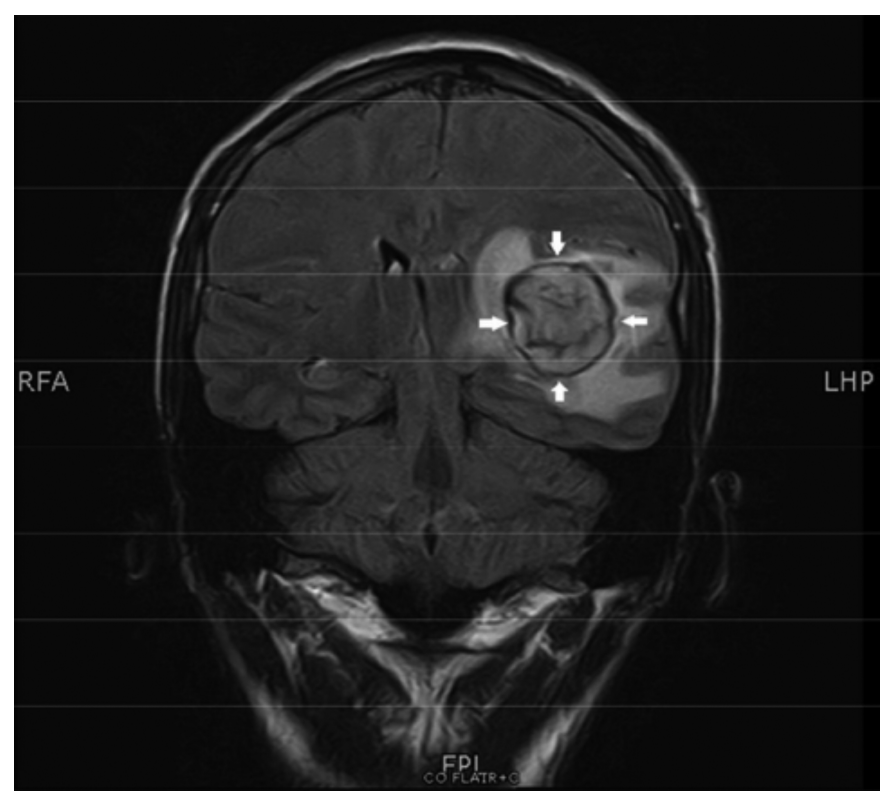

Figure 2: MRI brain FLAIR sequence coronal view. Brain abscess with surrounding oedema in left temporo-parietal lobe.
Lumbar puncture was not attempted due to space occupying lesion in brain on MRI. Transthoracic echocardiogram was done to rule out endocarditis, and showed visceroatrial situs solitus with dextrocardia without any evidence of vegetation. Patient was admitted in high dependency unit. Intravenous hydration with normal saline was started. He was given paracetamol intravenously on need basis for pain control and was empirically started on ceftriaxone 2000 mg twice daily intravenously.

When MRI brain revealed brain abscess, neurosurgery team was taken on board for mini-craniotomy for drainage of abscess. About 30 ml thick pus was drained and sentfor culture and sensitivity, which turned out positive for lactococcus lactis sensitive to penicillin. Fungal and mycobacterial cultures were negative. Histopathology revealed brain parenchyma with an abscess cavity filled with fibrino-purulent exudate and necrotic debris surrounded by inflamed fibrous tissue and areas of reactive gliosis without an evidence of granuloma or malignancy. Periodic acid - Schiff with diastase (PAS-D) special stain was negative for fungal organisms. Patient's symptoms resolved after surgery, and he was discharged after one week.

\section{DISCUSSION}

Lactococcus lactis cremoris is an important organism used in processing dairy products such as cheese, yogurt and other milk products. ${ }^{1}$ It is a commensal of mucocutaneous surfaces of cattle and also found on plant surfaces; and is considered non-pathogenic for humans. However, some human infections have been reported. ${ }^{3,4}$

To our knowledge, very few cases of brain abscess have been reported in the literature..$^{7-9}$ In previously reported cases, the preceding events included sinusitis and a dental infection; and the treatment consisted of surgical drainage, coupled with antibiotics. There are no set guidelines for choice of antibiotics. One case was treated with meropenem and vancomycin. In such infections, where no set guidelines are available, culture and sensitivity best guides the therapy. ${ }^{7-9}$ The exact pathogenesis and possible predisposing factors for lactococcus lactis infections in humans are not fully understood. ${ }^{10}$ In some previous reports, ${ }^{8}$ its infection was associated with a history of exposure to unpasteurised dairy products, raw vegetable ingestion or immunodeficiency. As for the route of infection, history of dental caries and dental surgery have been reported in literature with isolation of lactococcuslactis cremoris, both from cerebellar abscess and oral cavity without bacteremia. ${ }^{9}$ Therefore, direct extension from oral cavity was considered to be the infection route. The dental history might be important to understand the lactococcuslactis cremoris infection route.

Our patient had a history of unpasteurised milk ingestion and history of earache; and possible sinusitis a few weeks before hospitalisation. Blood culture was negative. Although, at the time of admission, there were no clinical signs of otitis media, as he had been treated by general practitioner. Therefore, we assume, he may have had otitis media with translocation of bacteria to brain parenchyma; but the antibiotics, he used 
(azithromycin), did not treat abscess due to poor meningeal penetration. It was our assumption only, we did not have any concrete evidence to support this causality of otitis media causing brain abscess in this case. Additionally, our patient did not have dental caries or history of tooth extraction.

In summary, ingestion of unpasteurised milk can predispose to lactococcus lactis infection. Lactococcus lactis infection can occur in an immunocompetent host. Early intervention in lactococcus brain abscess can lead to complete recovery without any sequelae.

\section{CONFLICT OF INTEREST:}

The authors declared no conflict of interest.

\section{PATIENT'S CONSENT:}

Informed consent was obtained from the patient to publish the data concerning this case.

\section{AUTHORS' CONTRIBUTION:}

IA, KA: Wrote the initial manuscript.

IA: Revised the final manuscript.

HT: Collected patient's data.

MAA: Did the literature search.

All authors have substantial contribution in revising the manuscript critically for important intellectual content; gave final approval of the version to be published; and agreed to be accountable for all aspects of the work in ensuring that questions related to the accuracy or integrity of any part of the work are appropriately investigated and resolved.

\section{REFERENCES}

1. Song $A A$, In LLA, Lim SHE, Rahim RA. A review on lactococcus lactis: From food to factory. Microb Cell Fact 2017; 16(1):55.doi: 10.1186/s12934-017-0669-x.
2. Azizpour M, Hosseini SD, Jafari P, Akbary N. Lactococcus lactis: A new strategy for vaccination. Avicenna J Med Biotechnol 2017; 9(4):163-8.

3. Cavanagh D, Fitzgerald GF, McAuliffe O. From field to fermentation: The origins of Lactococcus lactis and its domestication to the dairy environment. Food Microbiol 2015; 47:45-61. doi: 10.1016/j.fm.2014.11.001.

4. Kim HS, Park DW, Youn YK, Jo YM, Kim JY, Song JY, et al. Liver abscess and empyema due to Lactococcus lactis cremoris. J Korean Med Sci 2010; 25(11):1669-7. doi: 10.3346/jkms.2010.25.11.1669.

5. Mansour B, Habib A, Asli N, Geffen Y, Miron D, Elias N. A case of infective endocarditis and pulmonary septic emboli caused by lactococcus lactis. Case Rep Pediatr 2016; 2016:1024054. doi: 10.1155/2016/1024054.

6. Hadjisymeou S, Loizou P, Kothari P. Lactococcus lactis cremoris infection: Not rare anymore? BMJ Case Rep 2013; 2013: pii: bcr2012008479. doi: 10.1136/bcr-2012-008479.

7. Akhaddar A, El Mostarchid B, Gazzaz M, Boucetta M. Cerebellar abscess due to lactococcus lactis. A new pathogen. Acta Neurochir (Wien) 2002; 144(3): 305-6. doi: 10.1007/s007010200041.

8. Feierabend D, Reichart R, Romeike B, Kalff R, Walter J. Cerebral abscess due to lactococcus lactis cremorisin a child after sinusitis. Clin Neurol Neurosurg 2013; 115(5): 614-6. doi: 10.1016/j.clineuro.2012.06.007.

9. Topçu Y, Akıncı G, Bayram E, and Hız S, Türkmen M. Brain abscess caused by lactococcus lactis cremorisin a child. Eur J Pediatr 2011; 170(12):1603-5. doi: 10.1007/s00431011-1586-3.

10. Campos R, Pérez B, Armengod L, Múñez E, Ramos A. Lactococcus lactis thyroid abscess in an immunocompetent patient. Endocrinol Nutr 2015; 62(4):204-6. doi: 10.1016/ j.endonu.2014.12.003. 